\title{
Erratum to: Cone Beam Computed Tomography (CBCT) in the Field of Interventional Oncology of the Liver
}

\author{
Blanche Bapst ${ }^{1} \cdot$ Matthieu Lagadec $^{1} \cdot$ Romain Breguet $^{4}$ • \\ Valérie Vilgrain ${ }^{1,2,3} \cdot$ Maxime Ronot $^{1,2,3}$
}

Published online: 1 August 2015

(C) Springer Science+Business Media New York and the Cardiovascular and Interventional Radiological Society of Europe (CIRSE) 2015

\section{Erratum to: Cardiovasc Intervent Radiol DOI: $10.1007 / \mathrm{s} 00270-015-1180-6$}

In the published article, it is indicated that the rotation duration and the X-ray time delay of the CBCT scanning are 8 and 6-9 s, respectively (Table 1) in the study by Paul et al. The correct values are 2.72 and 6 or $9 \mathrm{~s}$ (for global and segmental injection), respectively.

The online version of the original article can be found under doi:10.1007/s00270-015-1180-6.

Maxime Ronot

maxime.ronot@bjn.aphp.fr

Blanche Bapst

blanchebapst@hotmail.com

Matthieu Lagadec

matthieu.lagadec@bjn.aphp.fr

Romain Breguet

romain.breguet@hcuge.ch

Valérie Vilgrain

Valerie.vilgrain@bjn.aphp.fr

1 Department of Radiology, Beaujon Hospital, University Hospitals Paris Nord Val de Seine, Beaujon, 100 bd general Leclerc, 92118 Clichy, Hauts-de-Seine, France

2 University Paris Diderot, Sorbonne Paris Cité, Paris, France

3 INSERM U1149, Centre de Recherche Biomédicale BichatBeaujon, CRB3, Paris, France

4 University Hospital of Geneva, Geneva, Switzerland 